\title{
Rate of Administration
}

National Cancer Institute

\section{Source}

National Cancer Institute. Rate of Administration. NCI Thesaurus. Code C69281.

The speed at which an action is performed. 